Original Research Paper

\title{
Near Critical Carbon Dioxide Sorption Induced Crystallization in PET
}

\author{
Aversa Raffaella and Apicella Antonio \\ Advanced Materials Lab, Department of Architecture and Industrial Design, Second University of Naples, Aversa, Italy
}

Article history

Received: 13-06-2016

Revised: 15-06-2016

Accepted: 18-06-2016

Corresponding Author:

Aversa Raffaella

Advanced Materials Lab, Department of Architecture and Industrial Design, Second University of Naples, Aversa, Italy

Email: antonio.apicella@unina2.it

\begin{abstract}
Near critical carbon dioxide sorption equilibria at $50^{\circ} \mathrm{C}$ and pressures up to 40 atms are analyzed and interpreted on the basis of the multiple sorption mechanisms possible in a glassy amorphous and semi crystalline polymer. Varying the penetrant-polymer testing temperature and external pressure it is possible to meet the full range of possible sorption behavior, from ideal Fickian diffusion to limiting relaxation controlled kinetics. Low sorption levels of low-pressure gases are attained through an ordinary Fickian mechanism. At higher gas pressures, however, higher penetrant sorption levels induce polymer relaxation affecting the final sorption behavior. A highly interacting solvent, as Carbon Dioxide in near critical conditions, leads to anomalous sorption ranging from relaxation controlled Fickian diffusion to limiting Case II and diffusion controlled relaxation, at high solvent uptakes (sorption from high activity vapor or from liquid phases). Generally, swelling has been observed to be accompanied by a solvent induced crystallization as detected by means of High pressure Differential Scanning Calorimeter (DSC) and Wide Angle Xray Scattering (WAXS).
\end{abstract}

Keywords: PET, Carbon Dioxide, Anomalous Sorption, Solvent Induced Crystallization

\section{Introduction}

The possible multimodal sorption modes of a glassy polymer greatly influence the actual kinetic of sorption. As indicated in Fig. 1, glassy polymers exhibit a complex mass transfer behavior. Both concentration gradient controlled diffusion and relaxation controlled swelling contribute to the rate and extent of penetrant sorption. The physical phenomena that simultaneously occur are dissolution, diffusion, swelling and relaxation, together with deformation, stress buildup and crystallization in the matrix. A wide variety of effects can be therefore exhibited depending on the mutual polymer-penetrant affinity, on the temperature and on the penetrant activity. Varying the temperature and the penetrant activity it is possible to meet the full range of behavior, from ideal Fickian diffusion to limiting Case II (relaxation controlled), Fig. 1. Penetrant diffusion and solvent induced polymer relaxation and crystallization, in fact, are temperature activated processes characterized by significantly different activation energies (Sarti and Apicella, 1980, Nicolais et al., 1984). A change of the testing temperature, for example, will modify the relative contributions of ordinary diffusion and relaxation on the overall sorption behavior and crystalline morphology. The complex problems of anomalous transport include time-dependent boundary conditions and diffusion coefficients, polymer relaxation providing the rate determining transport step and polymer crazing and crystallization accompanying penetrant sorption.

Previous works have established the evidence of strong interactions between PET and some organic solvents (Mensitieri et al., 1995). In particular, near critical carbon dioxide is able to strongly interact with this thermoplastic matrix leading to swelling and plasticization (Koros and Paul, 1978). As consequence of these interactions, the penetrated solvent allows easier polymer chain movements of the amorphous parts leading to the formation of the lower free energy crystalline structure. This phenomenon known as Solvent Induced Crystallization (SINC) has been detected for carbon dioxide sorption in PET even for sample with high initial crystalline fraction. An analogous behavior has been widely described in literature for some organic solvents sorption in polycarbonate, PEEK and poly-ethyleneterephthalate (Mensitieri et al., 1990; 1993). 


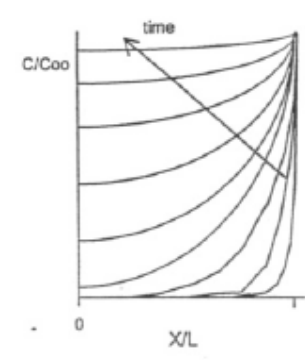

FCX 'S DIFFUSICN: MCOE I

$M \propto \sqrt{t}$

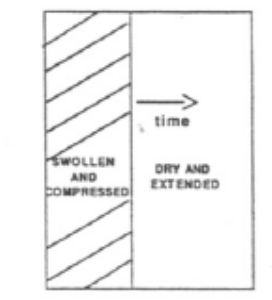

SWELLUNG AND RELAXATION: MOOE II

$M \propto \mathrm{t}$

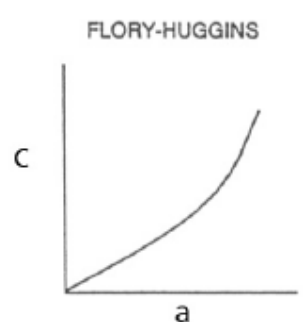

a ABSORPTION

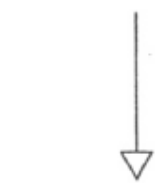

DUAL SORPTION MODEL

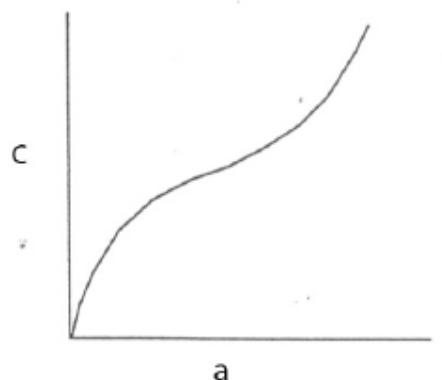

Fig. 1. Sorption modes

\section{Materials, Apparatus and Procedures}

\section{Materials}

High Molecular weight PET's of Intrinsic Viscosity of $0.85 \mathrm{dl} / \mathrm{g}$ have been used. Intrinsic viscosity has been measured at $25^{\circ} \mathrm{C}$ in phenol-ortodichlorobenzene $(60 / 40$ weight fraction) solutions. These measurements were made on $250 \mu \mathrm{m}$ amorphous PET films equilibrated at different pressures in gaseous Carbon dioxide at a temperature of $50^{\circ} \mathrm{C}$.

Reagent grade Carbon dioxide has been used. The Critical temperature and pressure were $31^{\circ} \mathrm{C}$ and 73.8 atms, respectively.

\section{Apparatus and Procedures}

\section{Thermal Analysis and Carbon Dioxide Sorption}

Differential Scanning Calorimetry: A DSC Mettler Differential Scanning Calorimeter equipped with a high pressure DSC27HP module has been used for the thermocalorimetric characterization. Samples films of $250 \mu \mathrm{m}$ have been used in the sorption/thermocalorimetric tests. Temperature scans from 0 to $300^{\circ} \mathrm{C}$ were carried out at $5^{\circ} \mathrm{C} / \mathrm{min}$.

A dual volume pressure decay apparatus (Mensitieri et al., 1995) has been used in the samples equilibration tests.
The modified DSC has been also used to determine the degree of gas uptakes and thermal induced crystallizations as described by Mensitieri et al. (1995). Specific isothermal tests at $50^{\circ} \mathrm{C}$ and increasing constant pressures $(3,6,9,13,16,21$ and 25 atms) were run in the modified calorimeters up to the gas equilibrium sorption. All DSC scans were made in Nitrogen atmosphere. A specifically design test was run maintaining the DSC cell at 40 atms of Carbon Dioxide during the thermal scan of a previously saturated in the same gas PET sample.

\section{Wide Angle X-Ray Scattering (WAXS)}

Wide-angle X-ray diffractograms have been obtained using a powder diffractometer PW 1050 Philips in a transmission arrangement on $250 \mathrm{~m}$ thick films. The $\mathrm{CuKa}$ radiation was used in the angular range $5^{\circ}-60^{\circ}$. Subtracting the amorphous halo from the original diffractogram between 50 and 400 and calculating by digital integration the respective area values evaluated the crystallinity amount.

\section{Results and Discussion}

The complex DSC traces of DSC scans run on samples previously conditioned up to equilibrium at increasing pressures are shown in Fig. 2. 


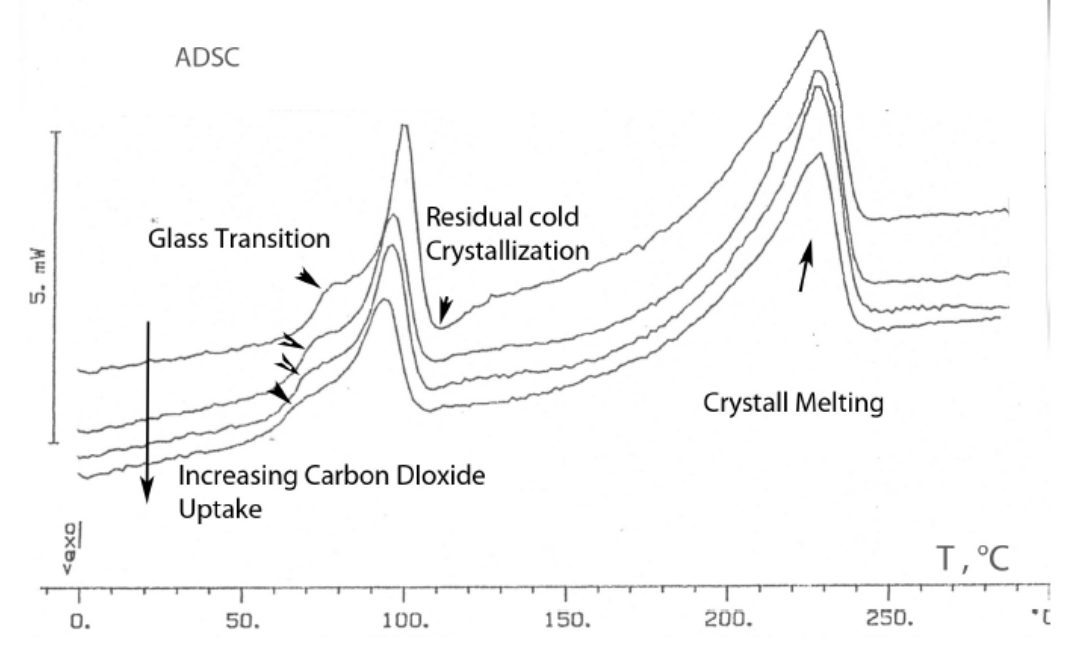

Fig. 2. DSC traces of samples conditioned in the modified DSC at 3, 6, 9 and $13 \mathrm{~atm}$

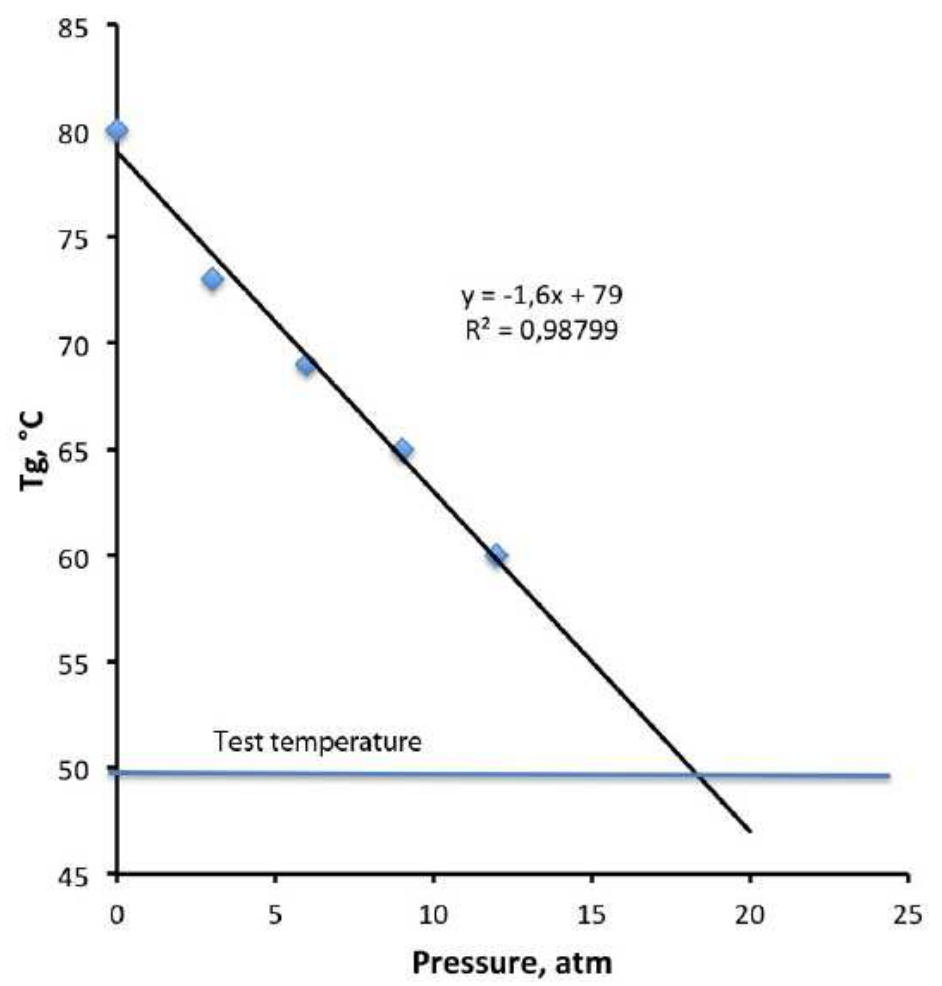

Fig. 3. Glass transition depressions for samples conditioned at 3, 6, 9 and 13 atms in the modified DSC

The first effect we have observed is that Carbon dioxide sorption at increasing pressures leads to evident glass transition depressions.

Moreover, a clear endothermic peak, which genesis will be discussed later, is observed just above the glass transition.

Finally a melting peak for all the samples, which is characteristic of the crystallized PET, is observed at $230-240^{\circ} \mathrm{C}$. This evidence clearly indicates that crystallization occurring in the $\mathrm{CO}_{2}$ conditioned and initially amorphous PET samples during Carbon dioxide equilibration and successive thermal scans. It could be hypothesized that cold crystallization, which generally occurs about at $165^{\circ} \mathrm{C}\left(85^{\circ} \mathrm{C}\right.$ above the unplasticized glass transition), is strongly favored by the presence of the sorbed Carbon dioxide.

Glass transition depressions due to Carbon dioxide PET plasticization are reported in Fig. 3. It could be 
inferred from the linear relationship between glass transition and Carbon dioxide equilibration pressure (linear regression $y=1.6 x+79$ with $\mathrm{R}^{2}=0.99$ ) that the glass transition of the samples saturated at 18-20 atms should reach a glass transition temperature that equals the testing temperature $\left(50^{\circ} \mathrm{C}\right)$. Equilibrium sorption uptakes measured in our PET samples conditioned at different pressures are shown in Fig. 4; the initial sorption behavior is characteristic of glassy polymers, but it is altered at pressures higher than $20 \mathrm{~atm}$. Swelling is hypothesized to occur when the glass transition of the plasticized PET reaches the test temperature $\left(50^{\circ} \mathrm{C}\right)$, namely when Carbon dioxide concentration in the polymer is higher than about 20 $\mathrm{cc}(\mathrm{STP}) / \mathrm{cc}$ at pressures above $20 \mathrm{~atm}$.

As previously discussed, the generally accepted mechanism, describing the transport of a penetrant in a polymeric material exposed to gas or vapor, is an activated solution-diffusion process (Holley et al., 1970).

Since sorption in glassy systems, as indicated in Fig. 1, is visualized as a process in which two modes are present, i.e., the penetrant molecules are either normally dissolved in the bulk polymer (FloryHuggins) and hence free to diffuse, or partially immobilized as in a sink (Languimir type), the kinetics of diffusion should be then also considered in order to correctly design the test (times and temperatures) accounting for the various mechanisms of sorption that can be active.

Penetrant sorption kinetic, in absence of significant polymer relaxation and accordingly to the dual sorption mode, should be described, as indicated before, by a combination of a Fick's law for the Flory-
Huggins dissolved species and an equivalent expression for the Langmuir adsorbed species that leads to a first limiting transport process characterized by smooth and continuous concentration profiles through the material and by an initially linear relationship between weight gain and square root of the exposure time (Crank, 2004). However, if the concentration of the penetrant is sufficiently high to swell and plasticize the polymer, a second limiting case called Case II (Holley et al., 1970), which is governed by relaxation controlled sorption mechanism, could be observed. Figure 5 resumes the previous findings describing the equilibrium sorption uptakes in PET below and above its glass transition; the curves relative to the rubber material and the initially glassy polymer coincide in the region of the near critical carbon dioxide state; this feature confirms that the initially glassy polymer is swollen and relaxes in the rubbery state as Carbon dioxide pressure overcomes a characteristic value for each temperature.

Carbon dioxide, hence, could act as a swelling solvent for this polymer when sorbed in its near critical conditions.

Above limiting concentration and in the supercritical condition, in fact, the Carbon dioxide plasticizes and swells to a rubbery state the PET. The rate of diffusion of the small and flexible Carbon dioxide molecules is rapid compared to that of other solvents even when the polymer is initially in the glassy state. However, when Case II sorption mechanism occurs, a swollen front separating a glassy un-penetrated core and the outer swollen shell is observed. This occurrence generates stresses and orientation at the interface.

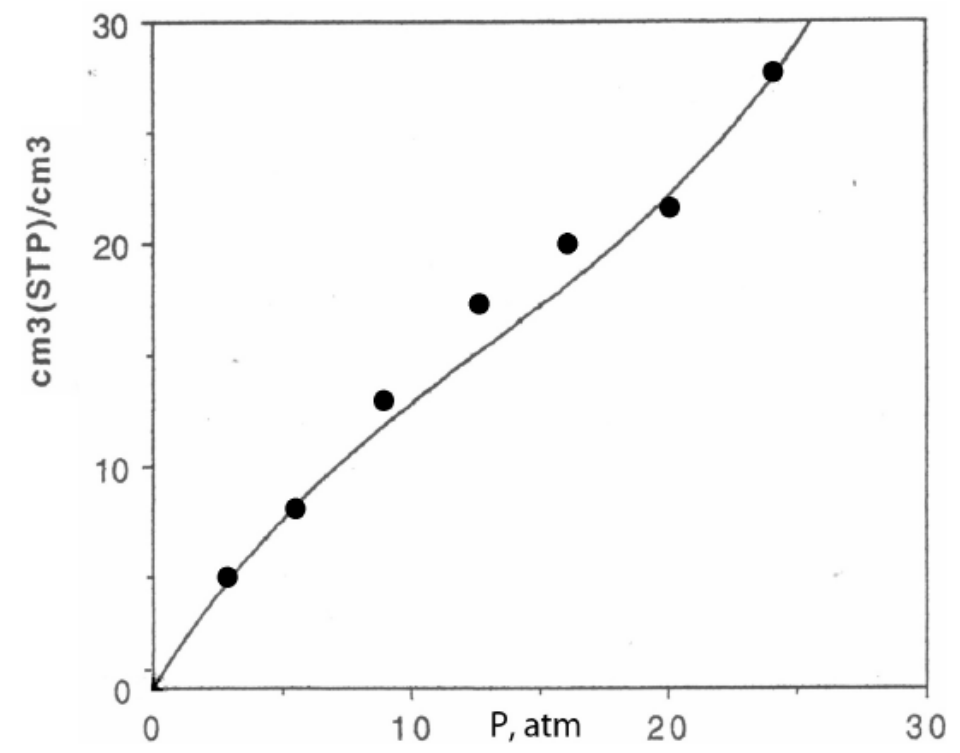

Fig. 4. Calorimetrically determined equilibrium Carbon dioxide sorption uptakes at $50^{\circ} \mathrm{C}$ in amorphous PET (Full dots exp. Full line, Koros et al., 1977) 


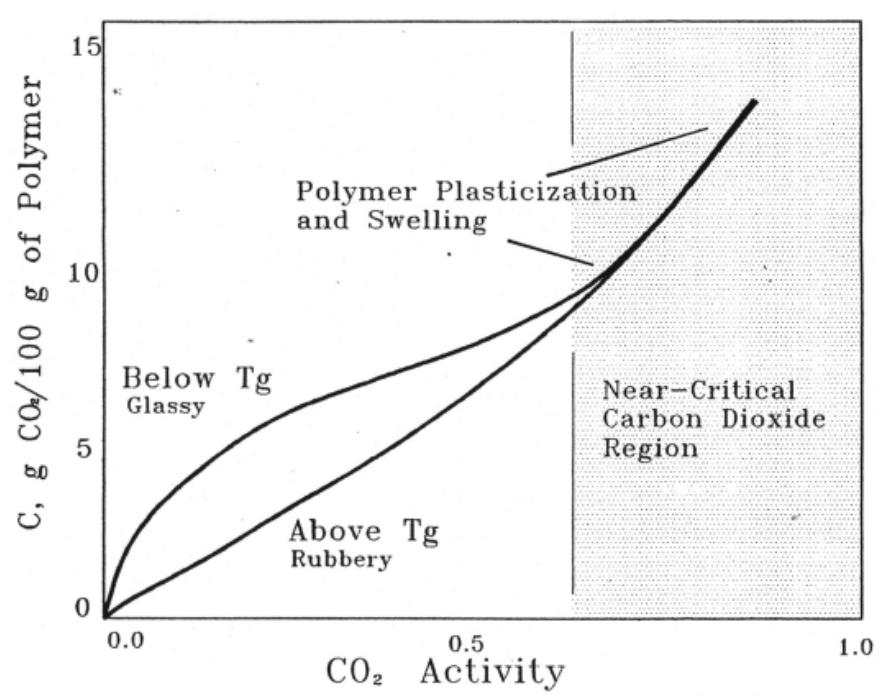

Fig. 5. Sorption uptakes in amorphous PET's in the glassy and rubbery states in the near critical Carbon dioxide

\section{Swelling Stresses, Relaxation Controlled Sorption and Crystallizations}

Solvent sorption can induce different levels of local swelling according to the amount of penetrant and to the intensity of the osmotic tensions generated by the solvent-polymer pair (Sarti and Apicella, 1980). The osmotic tension, po, differs from the more common osmotic pressure used for dilute polymer solutions in that can be calculated through polymer partial molar quantities (Sarti and Apicella, 1980). Osmotic tensions in swollen polymers, therefore, arise from the different solvent uptakes and swelling, between penetrated and un-penetrated polymer and can reach, depending on the distance from the glass transition, values of the order of tens of MPa (Sarti and Apicella, 1980).

The initially surface localized swelling tensions may promote, according to a characteristic mechanical craze or yield resistance of the polymer, a progressive swelling of the material which is characteristic of the so called "Case II" sorption. For this limiting second transport process, the weight gain and the discrete discontinuity between the solvent penetrated outer polymer shell and the central glassy core vary linearly with time when it is not controlle by the diffusion in the already penetrated and swollen polymer (Nicolais et al., 1984). This behavior, which is more often observed in thermoplastic polymers, is evident as solvent sorption uptake that linearly increases with time according to the constant rate penetration of a swollen and highly strained, advancing front. The rate of penetration of this front depends on the mechanical resistance of the polymer matrix and to its characteristic relaxation behavior (Sarti and Apicella, 1980).

The sorption of Carbon dioxide in PET films at our test temperature $\left(50^{\circ} \mathrm{C}\right)$ and pressures higher than 20 atms follows this type of sorption. The ratedetermining step is the osmotically induced polymer relaxation at the boundary between the unpenetrated glassy central core and the swollen outer shell (Sarti and Apicella, 1980). Because of the lower activation energy of the diffusive mechanism compared to that of relaxation, raising the test temperature, the polymer viscoelastic response to the Carbon dioxide osmotic stresses increases more than the diffusion rate, so that over a certain temperature the diffusive resistance becomes the controlling one (Nicolais et al., 1984; Apicella et al., 1993). In our case, however, diffusion rate of carbon dioxide is so high (Koros et al., 1977) that it is difficult to reach such controlling condition.

High pressure Case II sorption of Carbon dioxide sorption in PET is also characterized by Solvent INduced Crystallization (SINC).

The DSC scans reported in Fig. 2 shown the absence of a cold crystallization peaks, implying that, even at relatively low-pressure equilibration, the solvent treated sample reached high crystallinity level. Even if the quantitative evaluation of crystallinity fraction was not performed, values up to $25 \%$ have been expected.

Figure 6 reports a comparison between different DSC thermograms relative to the different treatment and testing procedure:

- The lower curve is relative to an amorphous PET sample

- The second curve is relative to a low temperature thermally crystallized sample

- The subsequent curve is relative to a solvent treated sample while

- The upper curve indicates the thermogram observed in a high pressure DSC temperature scan 
performed maintaining the saturated PET sample at 40 atms of $\mathrm{CO}_{2}$

Previous calorimetric investigations on similar systems (Mensitieri et al. 1995) reported that thermal treatments carried out at $100^{\circ} \mathrm{C}$ in absence of sorbed carbon dioxide needs very long times (upper curve relative to 15 days treatment) to induce level of crystallinity that are comparable to those obtained at $50^{\circ} \mathrm{C}$ and 40 atms after few minutes (lower curve in Fig. 2 and curve 3 in Fig. 6).

The presence of sorbing carbon dioxide lowers the onset of crystallization from about $150^{\circ} \mathrm{C}$ (see curve 1 in Fig. 6) to $90^{\circ} \mathrm{C}$ (see curve 4 in Fig. 6). Combination of Carbon dioxide sorption and thermal treatment favors the nucleation (which is thermodynamically favored at lower temperature, far from the melting temperature) and growth (which, conversely, is kinetically favored at higher temperatures) of crystallites in a range of temperatures where the two mechanisms do not interfere. In any type of crystallization, in fact, the mechanisms of nucleation and growth occur in a manner that specific crystallites size and morphology is determined. Table 1 reports the more common conditions and morphologies that can be obtained for PET.

The high Carbon dioxide uptakes indicates the great interaction with the polymer matrix that causes the swelling of the sample structure and the depression of the glass transition temperature to values lower than the test temperature $\left(50^{\circ} \mathrm{C}\right)$ leading to a Case II sorption accompanied by a SINC. The morphology of the spherulites formed in the plasticized polymer is such that very small crystallites are formed (nucleation is favored).

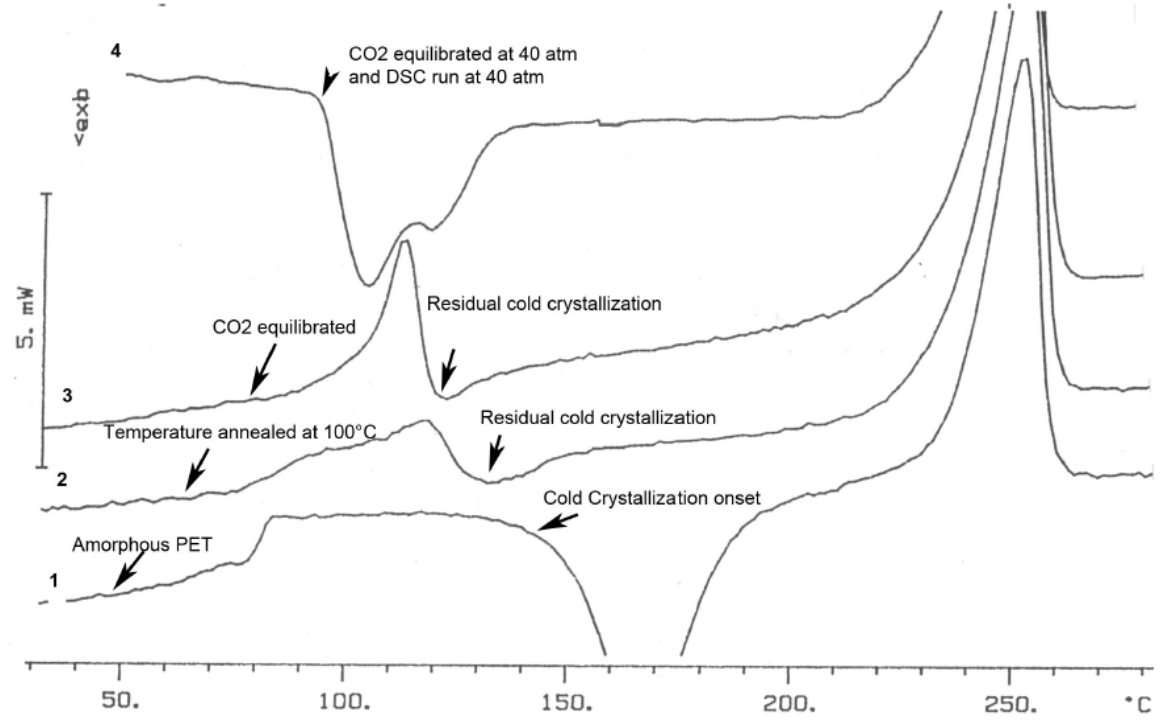

Fig. 6. DSC traces of thermal scans run on differently treated and crystallized PET samples

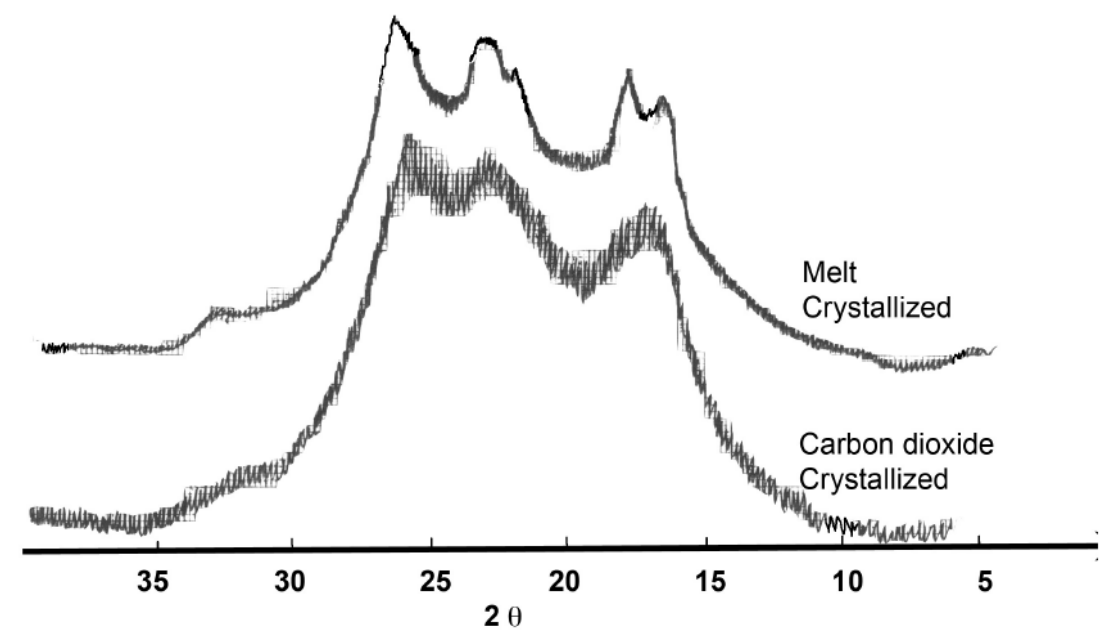

Fig. 7. Wide Angle X-ray Scattering (WAXS) run on differently treated and crystallized PET samples 
Table 1. Most common crystallization conditions and PET crystalline morphologies

\begin{tabular}{llll}
\hline Driving Force & Crystal Morphology & Temperature Range ${ }^{\circ} \mathrm{C}$ & State \\
\hline Thermal & Spherulites & $100-250$ & Molten \\
Strain Induced & Extended chain & $80-100$ & Near glassy \\
Shear Induced & Extended chain & $>250$ & Near melt \\
Solvent Induced & Spherulites & $<80$ & Near glassy \\
\hline
\end{tabular}

In Carbon dioxide sorption tests conducted at $50^{\circ} \mathrm{C}$ (Mensitieri et al., 1995), sorption uptake passes trough a maximum (overshoot). This phenomenon is associated to the process of solvent induced crystallization the squeeze out the previously adsorbed gas. A similar phenomenon occurs during the thermal scan reported in Fig. 2 and 6; the polymer, which is initially saturated with the penetrant that induced an extensive plasticization, undergoes further crystallization and recrystallization of existing solvent induced spherulites during the thermal scans that squeezes out and endothermally desorb the previously sorbed carbon dioxide.

The contribution of the endo desorption of the Carbon dioxide and the exo contribution of the crystallization lead to the complex behaviors reported above the glass transition in Fig. 2. It is interesting to notice that the DSC thermograms 3 and 4 of Fig. 6, which differ in the experimental procedures, result in different behavior above the sample glass transition.

Namely, WAXS measurements performed on samples as received and equilibrated in carbon dioxide are reported in Fig. 7.

WAXS analysis clearly confirms the presence of SINC with the formation of less perfect crystals compared to those obtained from the melt at higher temperatures.

The carbon dioxide treated samples readily show the onset of opacity due to the crystallization. Crystallization brings about the expulsion of solvent from developing crystallites; this process raises the local penetrant concentration and could lead to local saturation and phase separation (macro void formation). Nevertheless, in our case, the solvent expelled by the growing crystallites is accommodated inside the matrix reaching a concentration level higher than the true thermodynamic equilibrium value but without macro-void formation.

The out of equilibrium state of the polymer structure causes the onset of a mechanical matrix response that brings about the solvent desorption responsible of the maxima appearing in the sorption tests (Mensitieri et al., 1995). This matrix relaxation and consolidation phenomena are analogous to that detected in the case of some non crystallizing polymerdiluent systems (Holley et al., 1970).

\section{Conclusion}

The use of near critical gaseous environments leads the material to particular conditions of temperature that cannot be reached in other crystallization procedures.
Wide angle X-ray scattering has been used to demonstrate the onset of Solvent Induced Crystallization (SINC). Solvent uptake kinetic closely follows that of the Carbon dioxide Case II penetration front. Low pressure Carbon dioxide sorption has shown behaviors ranging from limiting Fickian diffusion to relaxation controlled diffusion depending on penetrant activities. Sorption from environment at increasing pressures has shown an increasing amount of penetrant concentration that induces crystallization above 20 $\mathrm{cm}^{3}(\mathrm{STP}) / \mathrm{cm}^{3}$ of polymer $16-20$ atms.

The crystalline form and the amount of crystallization that can be obtained will permit the investigation of the influence of micro-crystallinity in some of the mechanical and fatigue resistance of PET.

\section{Author's Contributions}

Prof. Aversa Raffaella, run all characterization tests and data interpretation. Prof. Apicella investigated on the base theoretical aspects.

\section{Ethics}

This article is original and contains unpublished material. The corresponding author confirms that all of the other authors have read and approved the manuscript and no ethical issues involved.

\section{References}

Apicella, A., B. Cappello, M.A. Del Nobile, M.I. La Rotonda and G. Mensitieri et al., 1993. Poly(Ethylene oxide) (PEO) and different molecular weight PEO blends monolithic devices for drug release. Biomaterials, 14: 83-90. DOI: 10.1016/0142-9612(93)90215-N

Crank, J., 2004. The Mathematics of Diffusion. 2nd Edn., Oxford Science Publications, England, ISBN-13: 978-0198534112.

Holley, R.H., H.B. Hopfenberg and V. Stannett, 1970. Anomalous transport of hydrocarbons in polystyrene. Polymer Eng. Sci., 10: 376-382. DOI: $10.1002 /$ pen.760100612

Koros, W.J., D.R. Paul, H.B. Hopfenberg, M. Fujii and V. Stannett, 1977. Effect of pressure on $\mathrm{CO}_{2}$ transport in poly(ethylene terephthalate). J. Applied Polymer Sci., 21: 2899-2904. DOI: 10.1002/app.1977.070211105 
Koros, W.J. and D.R. Paul, 1978. $\mathrm{CO}_{2}$ sorption in poly(ethylene terephthalate) above and below the glass transition. J. Polymer Sci. B, 16: 1947-1963. DOI: $10.1002 /$ pol.1978.180161105

Mensitieri, G., M.A. Del Nobile, A. Apicella, G. Guerra and H. Al Ghatta, 1995. Low temperature melting behavior of $\mathrm{CO}_{2}$ crystallized Modified PET's. Polymer Eng. Sci., 35: 506-512. DOI: $10.1002 /$ pen.760350608

Mensitieri, G., A. Apicella, M.A. Del Nobile and L. Nicolais, 1993. Extreme environmental resistance of PEEK matrix. J. Reinforced Plast. Comp., 12: 1138-1149. DOI: 10.1177/073168449301201101
Mensitieri, G., A. Apicella, M.A. Del Nobile, L. Nicolais and F. Garbassi, 1990. Solvent induced crystallization in poly(aryl-ether-ether-ketone). J. Mater. Sci., 25: 2963-2970.

DOI: $10.1007 / \mathrm{BF} 00584912$

Nicolais, L., A. Apicella and C. Denotaristefano, 1984. Timetemperature superposition of $n$-hexane sorption in polystyrene. J. Membrane Sci., 18: 187-196. DOI: $10.1016 / \mathrm{S} 0376-7388(00) 85033-4$

Sarti, G.C. and A. Apicella, 1980. Nonequilibrium glassy properties and their relevance in case II transport kinetics. Polymer, 21: 1031-1036.

DOI: 10.1016/0032-3861(80)90033-6 